\title{
Stable and scalable multistage terahertz-driven particle accelerator
}

\author{
Heng Tang ${ }^{1,2 *}$, Lingrong Zhao ${ }^{1,2 *}$, Pengfei Zhu ${ }^{1,2}$, Xiao Zou ${ }^{1,2}$, Jia $\mathrm{Qi}^{3}$, Ya Cheng ${ }^{3}$, \\ Jiaqi Qiu ${ }^{4}$, Xianggang $\mathrm{Hu}^{5}$, Wei Song ${ }^{5}$, Dao Xiang ${ }^{1,2,6,7 \dagger}$ and Jie Zhang ${ }^{1,2 \dagger}$ \\ ${ }^{1}$ Key Laboratory for Laser Plasmas (Ministry of Education), \\ School of Physics and Astronomy, \\ Shanghai Jiao Tong University, Shanghai 200240, China \\ 2 Collaborative Innovation Center of IFSA (CICIFSA), \\ Shanghai Jiao Tong University, Shanghai 200240, China \\ 3 State Key Laboratory of Precision Spectroscopy, \\ East China Normal University, Shanghai 200062, China \\ 4 Nuctech Company Limited, Beijing, 100084, China \\ 5 Science and Technology on High Power Microwave Laboratory, \\ Northwest Institute of Nuclear Technology, \\ Xi'an, Shanxi 710024, China \\ 6 Tsung-Dao Lee Institute, \\ Shanghai Jiao Tong University, Shanghai 200240, China \\ 7 Zhangjiang Institute for Advanced Study, \\ Shanghai Jiao Tong University, Shanghai 200240, China
}

(Dated: August 24, 2021)

\begin{abstract}
Particle accelerators that use electromagnetic fields to increase a charged particle's energy have greatly advanced the development of science and industry since invention. However, the enormous cost and size of conventional radio-frequency accelerators have limited their accessibility. Here we demonstrate a mini-accelerator powered by terahertz pulses with wavelengths 100 times shorter than radio-frequency pulses. By injecting a short relativistic electron bunch to a $30-\mathrm{mm}$-long dielectriclined waveguide and tuning the frequency of a 20-period terahertz pulse to the phase-velocitymatched value, precise and sustained acceleration for nearly $100 \%$ of the electrons is achieved with the beam energy spread essentially unchanged. Furthermore, by accurately controlling the phase of two terahertz pulses, the beam is stably accelerated successively in two dielectric waveguides with close to $100 \%$ charge coupling efficiency. Our results demonstrate stable and scalable beam acceleration in a multistage mini-accelerator and pave the way for functioning terahertz-driven highenergy accelerators.
\end{abstract}

Particle accelerators have played important roles in advancing science and industry, with applications ranging from high-energy colliders, synchrotron light sources, and free-electron lasers to x-ray imaging and cancer therapy machines. However, the large size and cost of conventional accelerators based on radio-frequency (rf) technology limit accessibility and are motivating development of compact accelerators [1 21. Among the techniques, terahertz $(\mathrm{THz})$-driven acceleration has great potential [15 21. Compared to the rf accelerator, the approximately 100-fold reduction in wavelength for the THz accelerator promises significant reduction in size, but increase in breakdown threshold to the gigaelectronvoltper-meter regime [22, 23]. Compared to the dielectric laser accelerator [13, 14, the approximately 100-fold increase in wavelength facilitates the fabrication of the accelerating structure and relaxes the tolerance on electron beam transverse size, pulse width, and timing for stable acceleration. Compared to plasma acceleration [1-12, the accelerating medium, i.e., dielectric-lined waveguide (DLW), is more stable and it is easier to control the beam size during staged acceleration.

Despite extensive efforts in the past few years, challenges, such as proof-of-principle $\mathrm{THz}$ acceleration to a functioning $\mathrm{THz}$ accelerator, remain. In a functioning accelerator, the beam is stably accelerated in successive modules to attain high energy since only finite energy gain can be reached in each accelerating structure. Therefore, a functioning $\mathrm{THz}$ accelerator requires stable whole-bunch acceleration, i.e., all the electrons in a bunch are accelerated at approximately the same phase to reach approximately the same energy, as well as high charge coupling efficiency and accurate phase control for staging. However, with the electron beam being longer than half of the $\mathrm{THz}$ wavelength, only a fraction of the electrons are accelerated by a laser-generated $\mathrm{THz}$ pulse, while others are decelerated [15-19]. Though recently, the staging of acceleration for relativistic electrons has been reported 21, the short acceleration length from an accelerator-produced single-cycle transition radiation $\mathrm{THz}$ pulse and the limited beam quality is not sufficient to address the question of whether an electron bunch can be stably accelerated in successive long structures with high charge coupling efficiency and high stability. We note that while whole-bunch acceleration for $\mathrm{keV}$ electron beam in two stages has been reported in [20], the small energy gain and large energy spread of the beam makes it difficult to answer the question of whether beam energy spread can be preserved in staged acceleration with considerable energy gain. 
(a)

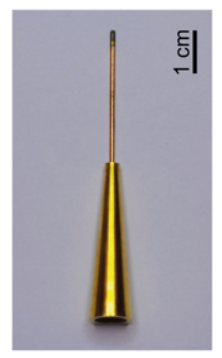

(c)

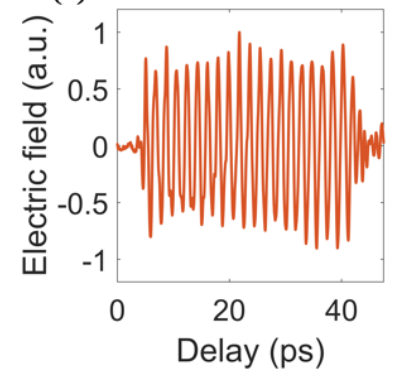

(b)

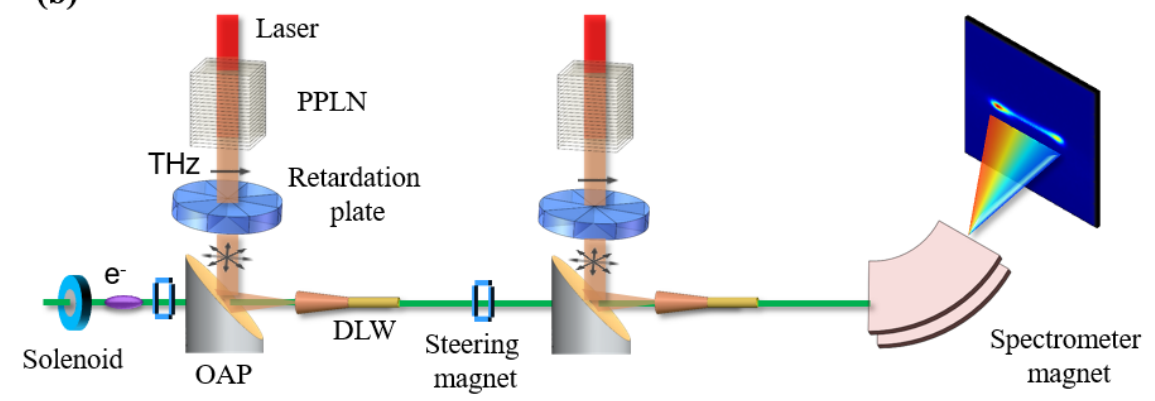

(d)

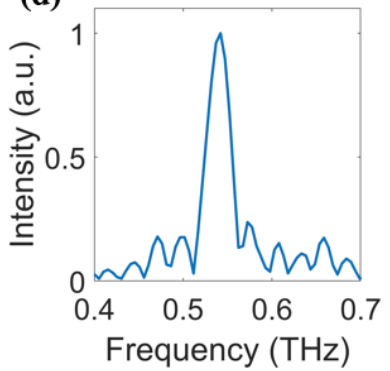

(e)

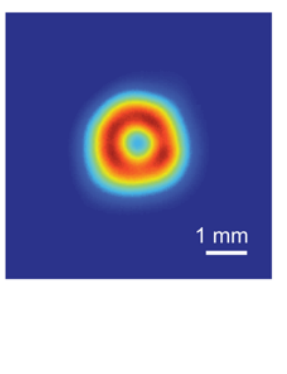

(f)

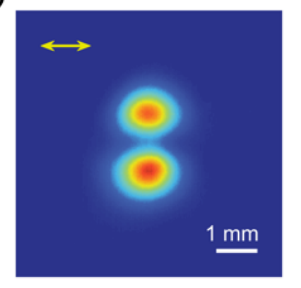

FIG. 1: Mini-accelerator powered by THz pulses. (a) Picture of DLW assembled with a horn; (b) Schematic of two-stage THz acceleration experiment; Measured $\mathrm{THz}$ waveform (c) and spectrum (d); Measured profiles of the radially polarized $\mathrm{THz}$ pulse before (e) and after (f) a polarizer (the arrow representing the direction of the wire).

In this Letter, we demonstrate sustained and stable whole-bunch acceleration of a relativistic electron beam in two stages driven by 20 -period $\mathrm{THz}$ pulses. The accelerating structure is a $30-\mathrm{mm}$-long DLW with $0.86 \mathrm{~mm}$ aperture, about 100 times smaller than a conventional rf structure. The DLW consists of a dielectric layer of fused silica with a wall thickness of $43 \mu \mathrm{m}$ and a goldcoated external surface. Our analysis shows that such a structure supports an accelerating mode at $0.54 \mathrm{THz}$ with a phase velocity of approximately $v_{p}=0.989 c$ and a group velocity of $v_{g}=0.72 c$ (see Supplementary Materials Fig.S1). A horn with an opening angle of 10 degrees is attached to the DLW to increase the coupling efficiency [15, 20, 21, which is measured to be about $70 \%$ in this experiment. An optical image of the mini-accelerator is shown in Fig. 1(a). The layout of the experiment is depicted in Fig. 1(b). A $3 \mathrm{MeV}, 10 \mathrm{fC}$ relativistic electron beam with a velocity of $v_{e}=v_{p}=0.989 c$, is successively accelerated in two identical modules consisting of an offaxis parabolic (OAP) mirror for focusing the $\mathrm{THz}$ pulse, a horn for adiabatically guiding the $\mathrm{THz}$ pulse, and a DLW for the THz-electron interaction. A solenoid is used to control the electron beam size and steering magnets are used to control the beam trajectory during staged acceleration. The beam energy distribution is measured with a spectrometer magnet (see Fig.S2).

The THz pulse is produced by sending a linearly polarized femtosecond laser (about $3 \mathrm{~mJ}$ ) through a cryogenically cooled periodically poled lithium niobate (PPLN) crystal 24 with a size of $3 \mathrm{~mm}$ by $3 \mathrm{~mm}$. The PPLN crystal has a total length of $4.4 \mathrm{~mm}$ and a period of 0.22 $\mathrm{mm}$ for producing a 20-period narrowband $\mathrm{THz}$ pulse centered around $0.54 \mathrm{THz}$. The $\mathrm{THz}$ waveform measured with electro-optic sampling is shown in Fig. 1(c), and the corresponding spectrum is shown in Fig. 1(d). The duration of the THz pulse is $\tau_{T H z}=40 \mathrm{ps}$, matching the length of the DLW for maximal energy gain. The temperature of the PPLN is reduced to below $200 \mathrm{~K}$ for reducing $\mathrm{THz}$ absorption. It should be noted that the frequency of the $\mathrm{THz}$ pulse depends on the refractive index of the laser and THz pulse in the crystal. Therefore, the THz pulse's central frequency may be varied by changing the crystal temperature, and in this experiment this offers about $6 \%$ tunability for the $\mathrm{THz}$ frequency while keeping the $\mathrm{THz}$ energy at a similar level (see Fig.S3).

To allow efficient excitation of the TM01 mode in DLW, the linearly polarized narrowband $\mathrm{THz}$ pulse is converted into a radially polarized pulse with a spatially variable retardation plate (see Fig.S4). This conversion ensures the best matching of the Poynting vector for optimally transferring energy from the incident $\mathrm{THz}$ pulse to the accelerating mode in the DLW. In general, the coupling efficiency in converting the energy of a $\mathrm{THz}$ pulse into that of a specific mode in the DLW depends on the similarity of the Poynting vector distribution. Therefore, a linearly polarized $\mathrm{THz}$ pulse can efficiently excite TM11-like deflecting mode 25] and a radially polarized $\mathrm{THz}$ pulse is required to excite the TM01 accelerating mode. The radially polarized $\mathrm{THz}$ pulse profile is measured with a $\mathrm{THz}$ camera and shows the expected donut shape (Fig. 1(e)). The THz energy is measured to be about $100 \mathrm{~nJ}$ at the entrance to the horn. It should be 
(a)
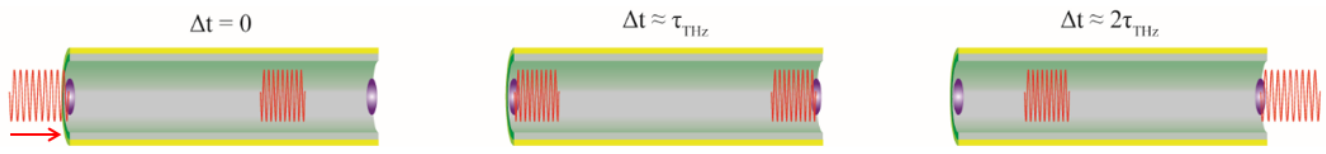

(b)

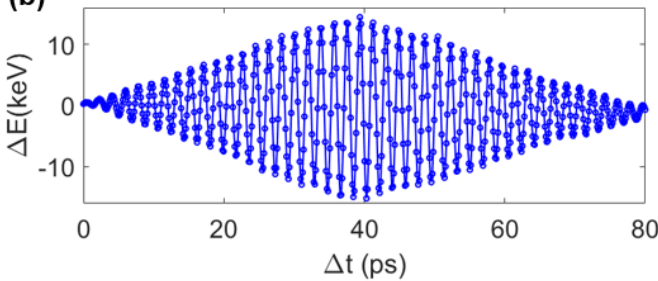

(c)

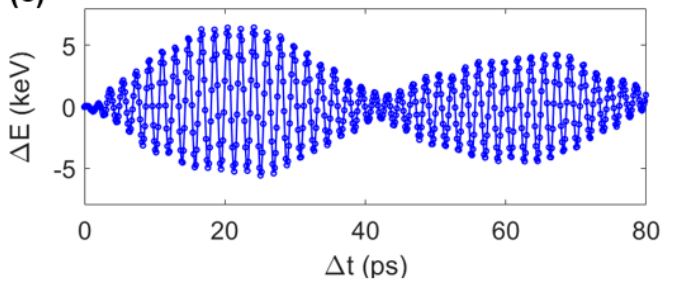

(d)

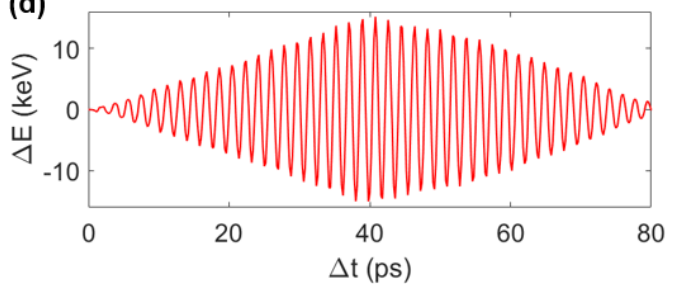

(e)

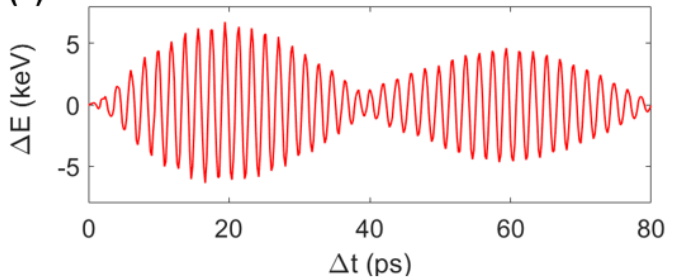

FIG. 2: Visualization of THz-electron interaction. (a) Schematic depiction of the relative position between the electron beam (purple) and $\mathrm{THz}$ pulse (red) at various time delays (the arrow represents the direction of travel); Measured electron energy changes when the $\mathrm{THz}$ frequency equals to (b) and deviates from (c) that of the accelerating mode; Simulated electron energy change when the $\mathrm{THz}$ frequency equals to (d) and deviates from (e) that of the accelerating mode.

noted that an azimuthally polarized pulse may also take a donut shape, so a wire grid polarizer is used to confirm the polarization. For a radially polarized pulse, the two lobes after the polarizer line up in the perpendicular direction of the wire (Fig. 1(f)), while those for an azimuthally polarized pulse should line up in the same direction of the wire (see Fig.S4).

In addition to exciting the accelerating mode, sustained acceleration also requires the $\mathrm{THz}$ phase velocity to be equal to the electron velocity, or, in other words, requires the $\mathrm{THz}$ frequency to be equal to that of the accelerating mode. In a conventional rf accelerator, this is achieved through precision machining of the rf cavity and utilization of a low-level rf system. Here we vary the crystal temperature to tune the frequency of the $\mathrm{THz}$ pulse in order to match that of the accelerating mode. With both the electron beam and $\mathrm{THz}$ pulse propagating in the same direction, this $\mathrm{THz}$ accelerator works in the traveling-wave mode, and the acceleration process can be visualized through measurement of beam energy change as a function of time delay $(\Delta t)$ between the $\mathrm{THz}$ pulse and electron beam.

To understand the acceleration process, we schematically depict the relative position of the electron and $\mathrm{THz}$ pulses at various time delays in Fig. 2(a). With the $\mathrm{THz}$ pulse frequency matching that of the accelerating mode, optimal acceleration should be achieved at $\Delta t=\tau_{T H z}=40 \mathrm{ps}$ when the electron beam catches up with the tail of the $\mathrm{THz}$ pulse at the entrance to the DLW. With the beam velocity higher than the group velocity of the $\mathrm{THz}$ pulse in the DLW, the electron beam interacts across the full $\mathrm{THz}$ envelope while remaining phase matched and achieves maximal energy exchange. Deviating from this time delay causes the beam to miss part of the $\mathrm{THz}$ pulse, and the energy exchange is reduced. This is exactly what has been observed in the experiment (Fig. 2(b)), where the envelope of the energy modulation monotonically increases for $\Delta t<40 \mathrm{ps}$ and then decreases for $\Delta t>40 \mathrm{ps}$. Within one wavelength window, the energy change depends on the delay time, which translates to the $\mathrm{THz}$ phase seen by the electron beam.

When the THz frequency deviates from that of the accelerating mode, the effective interaction length and energy exchange should be reduced (see Fig.S5). We tuned the $\mathrm{THz}$ frequency to $0.51 \mathrm{THz}$ (corresponding dephasing length, i.e. the distance over which the phase slippage increases to $\pi / 2$, is about $6.7 \mathrm{~mm}$ ) to see this effect, and the result is shown in Fig. 2(c). In contrast to the matching case, here at $\Delta t=40 \mathrm{ps}$, the energy exchange is close to zero. This is because the electron beam slips from the accelerating phase to the decelerating phase, and the energy gain from interaction with the first half of the $\mathrm{THz}$ pulse is canceled by the energy reduction from the second half of the THz pulse, similar to a detuned rf cavity. The simulated electron energy change for the matched and unmatched cases is in excellent agreement with the measurements shown in Fig. 2(d) and 2(e), respectively. Furthermore, no noticeable deflection to the beam has been measured, which indicates that most of the $\mathrm{THz}$ energy is converted into a single accelerating mode, demonstrating high efficiency in converting $\mathrm{THz}$ energy to ac- 
celerating mode and finally to the energy of the electron beam. Here an energy conversion efficiency of about 1.5 $\mathrm{keV}$ per $\mathrm{n} \mathrm{J}^{1 / 2}$ has been achieved, much higher than previous results (see Fig.S6). It also allows comparison and confirmation of predictive models and scaling laws and is critical for understanding the physics and engineering issues behind $\mathrm{THz}$ acceleration in long structures.

A functioning $\mathrm{THz}$ accelerator requires shot-to-shot stability and the staging of the accelerating structures for reaching higher energy. Here a second module $80 \mathrm{~cm}$ downstream of the first is used to demonstrate the feasibility, as well as the repeatability of the module. With similar tuning procedures, the energy gain of about 16 $\mathrm{keV}$ (for a $110 \mathrm{~nJ} \mathrm{THz}$ pulse) in the second DLW is achieved. We then vary the time delay of the second $\mathrm{THz}$ pulse to change the interaction phase (see Fig.S2), and the corresponding beam energy distribution, as well as transverse beam size (measured at a screen just before the spectrometer magnet), is shown in Fig. 3(a) and 3(b), respectively. From Fig. 3(a), one can see that when the electron bunch interacts with $\mathrm{THz}$ pulse at the on-crest phases, its energy is upshifted $(\phi=\pi / 2$, acceleration) or downshifted $(\phi=3 \pi / 2$, deceleration $)$ with minimal broadening. In contrast, at the zero-crossing phases $(0$ and $\pi$ ), the beam energy distribution receives maximal broadening with the centroid energy unchanged. Due to the initial beam energy chirp, the broadening at the two zero-crossing phases is slightly different (see Fig.S7). Similar to the zero-phasing method with rf structure [26] and laser streaking technique from attosecond metrology [27, electron's time information is mapped into its energy distribution when the interaction is around the zerocrossing phases. Accordingly, the electron bunch length is estimated to be about $100 \mathrm{fs}$ RMS and the beam arrival time jitter is determined to be about 50 fs RMS.

The beam size in Fig. 3(b) shows the effect of the transverse force in the DLW (see, e.g. 28]). Similar to an rf accelerator, the beam experiences a small focusing force at $\phi=\pi$ (bunch head is accelerated more than bunch tail) leading to a reduction in beam size, and a defocus-

(a)

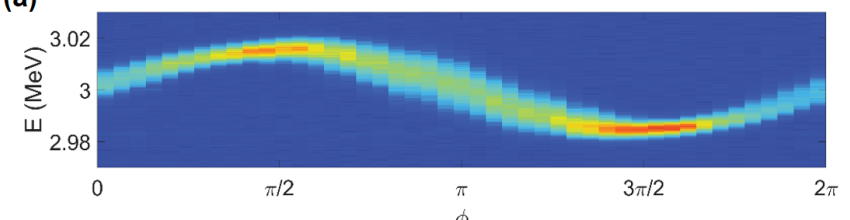

(b)

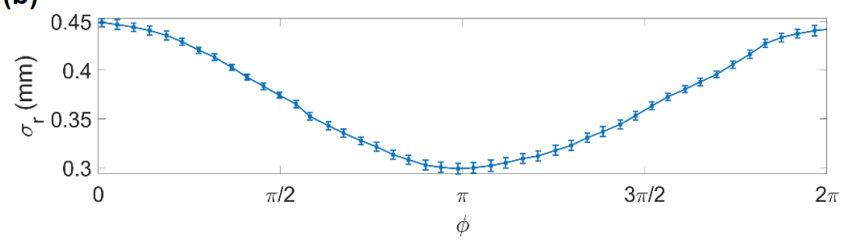

FIG. 3: Image of the electron beam on the spectrometer screen (a) and the transverse beam size (b) for various phases of the second $\mathrm{THz}$ pulse.

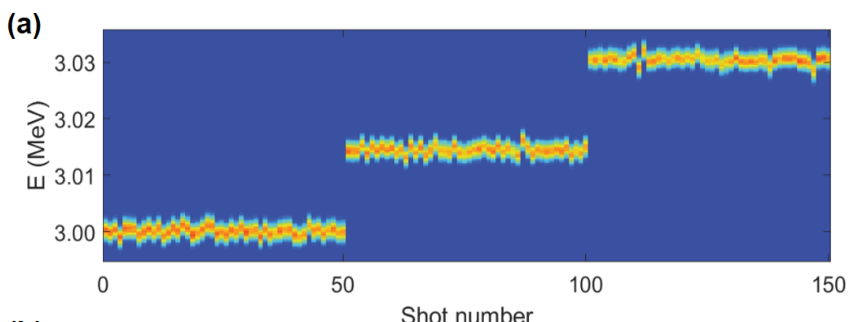

(b)

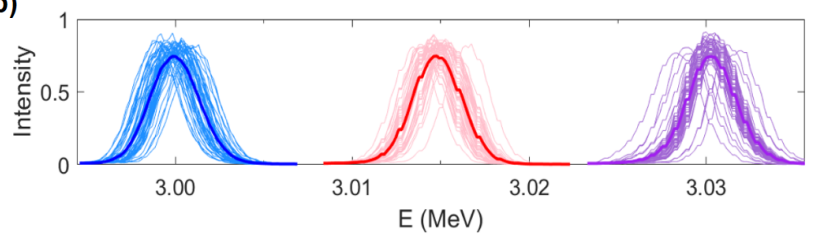

FIG. 4: Stable and staged THz-driven interaction. (a) Consecutive measurement of beam energy distribution with both $\mathrm{THz}$ pulses off (the first 50 shots), with one-stage acceleration (the middle 50 shots), and with two-stage acceleration (the final 50 shots); (b) Statistical results of the beam energy distribution for the data in (a).

ing force is exerted at $\phi=0$ where the bunch head is decelerated more, which is also the phase for bunch compression [29, 30]. The transverse force is negligibly small at the accelerating phase (electron beam size is similar to the case with $\mathrm{THz}$ off), which is crucial for maximizing the charge coupling efficiency in staging. Due to the absence of transverse force, nearly $100 \%$ charge coupling efficiency is achieved (see Fig.S8).

With the phases of the two $\mathrm{THz}$ pulses both set for maximal acceleration, consecutive measurements of the beam energy distribution after one-stage and two-stage acceleration are shown in Fig. 4(a). The corresponding statistical results for beam energy distribution are shown in Fig. 4(b). The beam energy spread (about 1.3 $\mathrm{keV}$ ) and energy jitter (about $0.6 \mathrm{keV}$ ) remain unchanged within the measurement accuracy after staged acceleration, setting a clear route to higher energy with more stages. The shot-to-shot fluctuation of the central beam energy is mainly from fluctuations of $\mathrm{rf}$ amplitude in the electron gun, and the fluctuation from the electron beam injection timing jitter is estimated to be about $0.2 \mathrm{keV}$.

In summary, we have demonstrated stable and scalable acceleration of a relativistic electron beam in successive DLWs powered by multicycle $\mathrm{THz}$ pulses. The energy gain achieved in each module of this mini-accelerator is about $15 \mathrm{keV}$, limited by the $\mathrm{THz}$ pulse energy (about $100 \mathrm{~nJ})$. First, we note that $\mathrm{keV}$ electrons have been widely used in ultrafast electron diffraction (UED) for probing nonequilibrium states and structural dynamics [31, 32. Our technique may immediately find applications in UED, e.g., enabling all-optical UED with matched or even enhanced performance. Second, the DLW may be used as an add-on module for compressing both relativistic and sub-relativistic beams [33 35], enhancing the performance of UED and 4D electron microscopy 36]. Third, narrowband $\mathrm{THz}$ pulse with 
millijoule-level energy has been demonstrated [37, so $\mathrm{MeV}$ energy gain could be obtained with such a $\mathrm{THz}$ source, which opens up new opportunities in fields as portable medical and industrial imaging, and cancer therapy. Furthermore, with the demonstrated stability and scalability, a functioning miniaturized high energy $\mathrm{THz}$ accelerator with dozens of stages can be foreseen.

This work was supported by the National Natural Science Foundation of China (Grants No. 11925505,
12005132,11504232 , and 11721091), the office of Science and Technology, Shanghai Municipal Government (No. 16DZ2260200 and 18JC1410700), and China National Postdoctoral Program for Innovative Talents (No. BX20200220).

* These authors contributed equally to this work. † dxiang@sjtu.edu.cn

† jzhang1@sjtu.edu.cn
[1] T. Tajima and J. Dawson, Laser electron accelerator, Phys. Rev. Lett. 43, 267 (1979).

[2] S. Mangles et al., Monoenergetic beams of relativistic electrons from intense laser-plasma interactions, Nature 431, 535 (2004).

[3] C. Geddes et al., High-quality electron beams from a laser wakefield accelerator using plasma-channel guiding, Nature 431, 538 (2004).

[4] J. Faure et al., A laser-plasma accelerator producing monoenergetic electron beams, Nature 431, 541 (2004).

[5] W. Leemans et al., GeV electron beams from a centimetre-scale accelerator, Nat. Phys. 2, 696 (2006).

[6] I. Blumenfeld et al., Energy doubling of $42 \mathrm{GeV}$ electrons in a metre-scale plasma wakefield accelerator, Nature 455, 741 (2007).

[7] X. Wang et al., Quasi-monoenergetic laser-plasma acceleration of electrons to $2 \mathrm{GeV}$, Nat. Commun. 4, 1988 (2013).

[8] W. Leemans et al., Multi-GeV electron beams from capillary-discharge-guided subpetawatt laser pulses in the self-trapping regime, Phys. Rev. Lett. 113, 245002 (2014).

[9] M. Litos et al., High-efficiency acceleration of an electron beam in a plasma wakefield accelerator, Nature 515, 92 (2014).

[10] S. Steinke et al., Multistage coupling of independent laserplasma accelerators, Nature 530, 190 (2016).

[11] E. Adli et al., Acceleration of electrons in the plasma wakefield of a proton bunch, Nature 561, 363 (2018).

[12] W. Leemans et al., Petawatt laser guiding and electron beam acceleration to $8 \mathrm{GeV}$ in a laser-heated capillary discharge waveguide, Phys. Rev. Lett. 122, 084801 (2019).

[13] E. Peralta et al., Demonstration of electron acceleration in a laser-driven dielectric microstructure, Nature 503, 91 (2013).

[14] N. Sapra et al., On-chip integrated laser-driven particle accelerator, Science 367, 79 (2020).

[15] E. Nanni et al., Terahertz-driven linear electron acceleration, Nat. Commun. 6, 8486 (2015).

[16] D. Zhang et al., Segmented terahertz electron accelerator and manipulator (STEAM), Nat. Photonics 12, 336 (2018).

[17] E. Curry, S. Fabbri, J. Maxson, P. Musumeci and A. Gover, Meter-scale terahertz-driven acceleration of a relativistic beam, Phys. Rev. Lett. 120, 094801 (2018).

[18] D. Zhang et al., Femtosecond phase control in high-field terahertz-driven ultrafast electron sources, Optica 6, 872 (2019).

[19] M. Hibberd et al., Acceleration of relativistic beams using laser-generated terahertz pulses, Nat. Photonics 14, 755
(2020).

[20] D. Zhang et al., Cascaded Multicycle Terahertz-Driven Ultrafast Electron Acceleration and Manipulation, Phys. Rev. X 10, 011067 (2020).

[21] H. Xu et al., Cascaded high-gradient terahertz-driven acceleration of relativistic electron beams, Nat. Photonics, https://doi.org/10.1038/s41566-021-00779-x (2021).

[22] M. Thompson et al., Breakdown limits on gigavolt-permeter electron-beam driven wakefields in dielectric structures, Phys. Rev. Lett. 100, 214801 (2008).

[23] B. O'Shea et al., Observation of acceleration and deceleration in gigaelectron-volt-per-metre gradient dielectric wakefield accelerators, Nat. Commun. 7, 12763 (2016).

[24] Y. Lee et al., Generation of narrow-band terahertz radiation via optical rectification of femtosecond pulses in periodically poled lithium niobate, Appl. Phys. Lett. 76, 2505 (2000).

[25] L. Zhao et al., Terahertz Oscilloscope for Recording Time Information of Ultrashort Electron Beams, Phys. Rev. Lett. 122, 144801 (2019).

[26] D. Wang, G. Krafft, and C. Sinclair, Measurement of femtosecond electron bunches using a rf zero-phasing method, Phys. Rev. E, 57, 2283 (1998).

[27] F. Krausz and M. Ivanov, Attosecond physics, Rev. Mod. Phys. 81, 163 (2009).

[28] C. Jing, Dielectric wakefield accelerators, Reviews of Accelerator Science and Technology. 9, 127 (2016).

[29] J. Maxson, D. Cesar, G. Calmasini, A. Ody, P. Musumeci, and D. Alesini, Direct measurement of sub$10 \mathrm{fs}$ relativistic electron beams with ultralow emittance, Phys. Rev. Lett. 118, 154802 (2017).

[30] L. Zhao et al., Terahertz Streaking of Few-Femtosecond Relativistic Electron Beams, Phys. Rev. X, 8, 021061 (2018).

[31] H. Ihee et al., Direct Imaging of Transient Molecular Structures with Ultrafast Diffraction, Science 291, 458 (2001).

[32] B. Siwick, J. Dwyer, R. Jordan and R. Dwayne Miller, An atomic-level view of melting using femtosecond electron diffraction, Science 302, 1382 (2003).

[33] C. Kealhofer, W. Schneider, D. Ehberger, A. Ryabov, F. Krausz and P. Baum, All-optical control and metrology of electron pulses, Science 352, 429-433 (2016).

[34] L. Zhao et al., Femtosecond relativistic electron beam with reduced timing jitter from THz-driven beam compression, Phys. Rev. Lett, 124, 054802 (2020).

[35] E. Snively et al., Femtosecond Compression Dynamics and Timing Jitter Suppression in a THz-driven Electron Bunch Compressor, Phys. Rev. Lett, 124, 054801 (2020). 
[36] A. Zewail, Four-dimensional electron microscopy, Science 328, 187 (2010).

[37] S. Jolly et al., Spectral phase control of interfering chirped pulses for high-energy narrowband terahertz generation, Nat. Commun. 10, 2591 (2019). 